\title{
PROPUESTAS PARA EL DESARROLLO DE LA INTERCULTURALIDAD EN LA EDUCACIÓN EN EL TIEMPO LIBRE DE BASE COMUNITARIA EN CATALUNYA
}

\author{
PROPOSALS FOR THE DEVELOPMENT OF INTERCULTURALITY \\ IN EDUCATIONAL PROGRAMMES CARRIED OUT DURING LEISURE TIME \\ AND WITH A COMMUNITY APPROACH IN CATALONIA \\ PROPOSTA PARA O DESENVOLVIMENTO DA INTERCULTURALIDADE \\ NA EDUCAÇÃO EM TEMPO LIVRE DE BASE COMUNITÁRIA NA CATALUNHA
}

\author{
Edgar IGLESIAS-VIDAL \& Berta ESPONA-BARCONS
}

Universidad Autónoma de Barcelona (UAB), Grupo de Investigación ERDISC

\begin{tabular}{|c|c|}
\hline & $\begin{array}{r}\text { Fecha de recepción del artículo: 10.VI.2016 } \\
\text { Fecha de revisión del artículo: 12.XII.2016 } \\
\text { Fecha de aceptación final: } 17.1 .2017\end{array}$ \\
\hline $\begin{array}{l}\text { PALABRAS CLAVE: } \\
\text { educación } \\
\text { tiempo libre } \\
\text { programas } \\
\text { interculturales } \\
\text { segregación } \\
\text { equidad educativa }\end{array}$ & $\begin{array}{l}\text { RESUMEN: La educación en el tiempo libre de base comunitaria ha jugado un papel impor- } \\
\text { tante en la construcción de la cohesión social en Catalunya. En este artículo se pone en valor } \\
\text { esta contribución, pero a su vez se señala como problemática la existencia de procesos de } \\
\text { concentración y segregación educativa que pueden constituir un obstáculo para la intercultu- } \\
\text { ralidad. Se presentan propuestas que ayuden a plantear, desde estas organizaciones, una ges- } \\
\text { tión intercultural de sus proyectos socioeducativos, por ejemplo, reflejando en ellos mayores } \\
\text { cuotas de diversidad sociocultural. MÉTODO. La investigación tenía como finalidad contribuir } \\
\text { al fortalecimiento de interculturalidad en este contexto, a partir de los siguientes objetivos: } \\
\text { identificar condiciones organizativas y educativas que favorecieran o dificultaran el desarrollo } \\
\text { de la interculturalidad; y, formular propuestas para su sostenibilidad o superación. Se aplica- } \\
\text { ron diferentes técnicas cualitativas para la recogida de datos: análisis documental, entrevistas } \\
\text { semi-estructuradas y grupos de discusión. Para el tratamiento y análisis de los datos se realizó } \\
\text { un análisis crítico del discurso y se utilizó el programa Atlas-Ti. RESULTADOS. Los resulta- } \\
\text { dos obtenidos constatan que, aunque coexisten condiciones favorables y desfavorables a la } \\
\text { interculturalidad, tienen más peso aquellas que limitan la diversidad sociocultural entre sus } \\
\text { participantes, y que los equipos educativos no tienen desarrollada, o muy poco, la dimensión } \\
\text { comunitaria. DISCUSIÓN. Partiendo de los resultados y de la literatura revisada se propone, } \\
\text { por un lado, impulsar medidas organizativas orientadas a la participación, la atención a la }\end{array}$ \\
\hline
\end{tabular}

CONTACTAR CON LOS AUTORES: Edgar Iglesias-Vidal. Universidad Autónoma de Barcelona (UAB), Departament de Pedagogia Aplicada. Edifici G6-255. Campus de Bellaterra. 08193, Bellaterra (Cerdanyola del Vallès). Grupo de Investigación ERDISC. Edgar. iglesias@uab.cat. 


\begin{tabular}{|c|c|}
\hline & $\begin{array}{l}\text { diversidad y la formación. Por otro, diseñar e impulsar acciones conjuntas con la comunidad } \\
\text { educativa para gestionar la existencia de sobre-representaciones de perfiles socioculturales } \\
\text { entre los participantes. Y por último, se sugiere la constitución de equipos comunitarios de } \\
\text { atención a la diversidad que promuevan una gestión intercultural compartida para superar los } \\
\text { procesos de concentración y segregación educativa. }\end{array}$ \\
\hline $\begin{array}{l}\text { KEY WORDS: } \\
\text { education } \\
\text { leisure time } \\
\text { intercultural } \\
\quad \text { programs } \\
\text { segregation } \\
\text { educational equity }\end{array}$ & $\begin{array}{l}\text { ABSTRACT: INTRODUCTION. The educational programmes carried out during leisure time } \\
\text { and with a community approach played an important role in the development of social cohe- } \\
\text { sion in Catalonia. In this paper the value of this contribution is recognized, but at the same } \\
\text { time, the existence of educational concentration and segregation dynamics is identified as a } \\
\text { problem for interculturality. For this reason, some proposals presented here can help these } \\
\text { organizations to manage interculturally their projects, for example, reflecting the sociocultur- } \\
\text { al diversity of the community within they are developed. METHOD. The framework of this } \\
\text { paper is a research project oriented to contribute to the strengthening of intercultural vision } \\
\text { in this context. The goals were: identify organizational and educational conditions which fa- } \\
\text { cilitate or not the development of interculturality; and, make proposals for their sustainability } \\
\text { or improvement. Some qualitative technics were used to collect data: documentary analysis, } \\
\text { semi-structured interviews and discussion groups. And Atlas-Ti program, as well as, discourse } \\
\text { critical analysis procedure were used to analyse the data. RESULTS. The results pointed out } \\
\text { that, even though there are advantageous and disadvantageous conditions to interculturality, } \\
\text { those that restrict the sociocultural diversity of participants influence more. And it was con- } \\
\text { firmed that educational teams have not developed, or very little, their community dimension. } \\
\text { DISCUSSION. On the basis of the results obtained and the literature reviewed is suggested, } \\
\text { on one hand, the impulse of some organizational actions oriented to participation, diversi- } \\
\text { ty attention and training actions. On the other, it is noted the appropriateness of designing } \\
\text { and launching actions, together with the educational community, to manage the existence of } \\
\text { over-representation of some sociocultural profiles among participants, and finally, it is pro- } \\
\text { posed the constitution of diversity attention' community teams to manage interculturality } \\
\text { among educational community and oriented to overcome the concentration and segregation } \\
\text { educational dynamics. }\end{array}$ \\
\hline $\begin{array}{l}\text { PALAVRAS-CHAVE: } \\
\text { educação } \\
\text { tempo livre } \\
\text { programas } \\
\quad \text { interculturais } \\
\text { segregação } \\
\text { equidade educativa }\end{array}$ & $\begin{array}{l}\text { RESUMO: INTRODUÇÃO. A educação em tempo livre de base comunitária teve um papel } \\
\text { importante na construção da coesão social na Catalunha. Neste artigo o valor dessa contri- } \\
\text { buição se faz presente, mas também sinaliza como problemática a existência de processos } \\
\text { de concentração e segregação educativa que podem constituir um obstáculo para a intercul- } \\
\text { tural idade. MÉTODO. A pesquisa em que se situa este artigo teve como finalidade contri- } \\
\text { buir ao fortalecimento da interculturalidade nesse contexto. Seus objetivos eram: identificar } \\
\text { condições organizativas e educativas que favoreceram ou dificultaram o desenvolvimento da } \\
\text { interculturalidade; e, formular propostas para a sua sustentabilidade ou superação. Foram } \\
\text { aplicadas diferentes técnicas qualitativas para a recolhida de dados: análises documentárias, } \\
\text { entrevistas semiestruturadas e grupos de discussão. E para o tratamento e análises dos dados } \\
\text { foi realizada uma análise crítica do discurso e a utilização de um programa Atlas-Ti. RESUL- } \\
\text { TADOS. Os resultados alcançados constataram que, ainda que existam condições favoráveis } \\
\text { e desfavoráveis à interculturalidade, têm mais peso aquelas que limitam a diversidade socio- } \\
\text { cultural entre seus participantes, além de que as equipes educativas não desenvolveram, ou } \\
\text { desenvolveram muito pouco, a dimensão comunitária. DISCUSSÃO. Partindo dos resultados } \\
\text { e da literatura revisada propõem-se, por um lado, impulsar medidas organizativas orientadas } \\
\text { à participação, atenção à diversidade e formação. Por outro lado, desenhar e impulsar ações } \\
\text { conjuntas com a comunidade educativa para gestionar a existência de sobre-representações } \\
\text { de perfis socioculturais entre os participantes. E por último, se sugere a constituição de equi- } \\
\text { pes comunitárias de atenção à diversidade, que promovam uma gestão intercultural compar- } \\
\text { tilhada, com o fim de superar os processos de concentração e segregação educativa. }\end{array}$ \\
\hline
\end{tabular}

\section{Introducción}

En este apartado se exponen, en primer lugar, algunas ideas sobre el papel que ha jugado la educación en el tiempo libre de base comunitaria en la construcción de la cohesión social en Catalunya. Ello permite analizar fortalezas y debilidades de estos proyectos socioeducativos en lo que a inclusión de la diversidad sociocultural se refiere. $\mathrm{Y}$, en segundo lugar, se aborda, a nivel conceptual, la necesidad de reflejar mayores cuotas de diversidad sociocultural como condición para una mayor interculturalidad en este ámbito educativo.

\section{La educación en el tiempo libre de base comunitaria como elemento clave para la cohesión social}

En los últimos años la educación en el tiempo libre en Catalunya ha contribuido significativamente a la cohesión social desde la implicación comunitaria en los contextos locales donde actúa. 
Son varias las aportaciones que así lo señalan (Essomba, 2009; Vallory, 2010) y que destacan que, si bien existen diferentes formas organizativas en este ámbito educativo, todas tienen en común un método pedagógico y una axiología crítica coherente con la igualdad, la participación y la transformación social.

Este contexto también se caracteriza por su intencionalidad educativa y su capacidad de generar entre los participantes fuertes vínculos afectivos. Estos lazos suelen traspasar el espacio de las organizaciones en los que se crearon. Por todo ello, la experiencia de la participación se define como positiva e intensa, y sus participantes suelen identificarse afectivamente con el proyecto educativo de la organización.

En los últimos años se ha puesto en valor la base comunitaria del tiempo libre (Generalitat de Catalunya, 2010). Éste es un elemento descriptivo y diferenciador en relación a otras propuestas educativas existentes en el mismo ámbito, pero que no han desarrollado sus proyectos con y para la comunidad, ni con una perspectiva inclusiva.

Por ello, la educación en el tiempo libre de base comunitaria (de ahora en adelante ETLBC) debe ser entendida como un contexto educativo estratégico para la cohesión social. El principal motivo es que puede posibilitar, desde sus proyectos educativos, que toda la diversidad sociocultural presente en los territorios locales de acción interactúe. También, y desde su relación con los centros educativos, puede contribuir a disminuir indicadores de riesgo educativo, tal y como indican trabajos como los de Anderson et al. (2014) en California.

No obstante, resulta conveniente evidenciar la existencia en la ETLBC de una tensión entre dos lógicas de naturaleza opuesta. Por un lado, el compromiso con la transformación social y la inclusión educativa, y por otro, las desigualdades para el acceso y participación del conjunto de la diversidad sociocultural en sus proyectos educativos.

Recientemente se señalaba (Fundació Ferrer i Guardia, 2013) que, en Catalunya, únicamente el $1,5 \%$ de las familias de los participantes en los esplais son de origen extranjero. Y, por otra parte, una referencia situada en el ámbito de los Centros Abiertos' (Xarxa de Centres Oberts de Barcelona, s.f.) definía como deseable que la mitad de sus participantes no presentase situaciones de exclusión o vulnerabilidad socioeconómica, ya que se corría el riesgo de la estigmatización socioeducativa. Así pues, aunque inicialmente se apueste por la diversificación de perfiles, tanto desde el punto de vista de la condición social como del origen cultural, la realidad indica que se supera la proporción señalada como deseable. Aun así, un informe reciente señalaba que:

Si bien la educación en el tiempo libre es un importante ámbito de reproducción de las desigualdades sociales, cabe decir que, gracias a la función de la iniciativa social, con el apoyo de las administraciones públicas, también es un ámbito fuertemente comprometido con la equidad y la comprensión de estas desigualdades (Síndic de Greuges, 2014, p. 11).

Sin duda la gestión y superación de estas desigualdades por parte de los equipos educativos supone un gran reto, pues son varios los ámbitos que contribuyen a la conformación de estas lógicas de exclusión educativa. En los últimos años, estos equipos educativos han ido desarrollando medidas organizativas para un mayor acceso de la diversidad sociocultural. Por ejemplo: facilidades de pago para familias con dificultades económicas; o comunicación del proyecto educativo mediante estrategias comunicativas (como trípticos explicativos) a grupos socioculturales recientemente establecidos en la comunidad. No obstante, y en la práctica, no han resultado del todo eficientes. $Y$, además, existen otros elementos que se originan fuera del ámbito de decisión y gestión de los mismos equipos y que promueven lógicas de exclusión, como pueden ser la legislación o las reducciones presupuestarias en el ámbito público.

Internacionalmente son varias las voces que reclaman un espacio propio de este ámbito educativo como palanca para la activación de procesos de inclusión educativa:

Inclusive leisure education programs are needed that embrace all human beings, especially individuals and groups who have been oppressed and encounter leisure constraints relative to their gender, race, ethnicity, ability, age, sexual orientation, income, living situation, religion, and other salient characteristics $^{2}$. (American Association for Physical Activity and Recreation [AAPAR], 2011, p. 44).

$Y$ finalmente, cabe destacar que la ETLBC resulta un contexto de estudio poco abordado desde una perspectiva de análisis global que permita profundizar en la participación de la diversidad sociocultural en estos espacios. No existen mecanismos desde los que se sistematicen este tipo de datos, y las aproximaciones que se han realizado en forma de estudio, aunque útiles e indicativas, se enfocan desde una visión sectorizada y no global de la ETLBC (Fundació Ferrer i Guardia, 2013). 


\section{La representación de la diversidad sociocultural entre los y las participantes como elemento clave para la interculturalidad}

La perspectiva intercultural ofrece un valioso marco teórico y de acción en coherencia con el principio de inclusión educativa y con el fin de contar con mayores cuotas de diversidad sociocultural entre los participantes. A continuación, se presentan algunos fundamentos interculturales $y$ se relacionan con las implicaciones prácticas que pueden tener para la ETLBC:

- ¿Para qué se construye la interculturalidad? La construcción de la cohesión social es una finalidad intercultural, y para ello deben superarse las condiciones que generen exclusión. También debe reconocerse el carácter axiológico y no exento de neutralidad del proyecto intercultural (Abdallah-Pretceille, 2006; Bartolomé, 2002; Wood \& Landry, 2008). Así, en el contexto de la ETLBC, la perspectiva intercultural se concreta identificando y superando los factores que, desde una perspectiva de equidad educativa y en un contexto de diversidad, dificultan el acceso de las minorías socioculturales a los proyectos educativos.

- ¿Cómo se construye? Los ejes igualdad y reconocimiento son básicos para el desarrollo de la interculturalidad. Para ello se requiere que las relaciones que se establezcan se den en condiciones de igualdad, asumiendo que el conjunto de significados y aportaciones cuenten con la misma validez (Giménez, 2012). Así, en la ETLBC, la interculturalidad se construye libre de jerarquías, estableciendo relaciones fruto de la participación que suponen una experiencia positiva para el conjunto.

- ¿Cuándo se construye? La interculturalidad se concibe como un proceso en permanente construcción, no siendo un objetivo propiamente y sí un instrumento para logros estratégicos como la cohesión social (Sales, 2011; Zapata-Barrero \& Pinyol, 2013). Por ello, desde la ELTBC, se cuenta con la capacidad de generar relaciones personales entre sus participantes sostenibles en el tiempo, que fácilmente trascienden la geografía física de la organización. Además, así se contribuye desde el presente a la construcción de la ciudadanía del futuro.

- ¿Quiénes son los participantes? La interculturalidad queda contextualizada desde el principio de inclusión educativa (Essomba, 2011), y para su desarrollo se necesita al conjunto de la diversidad (Consejo de Europa, 2008). Así, desde la ETLBC, resulta indispensable reflejar entre sus participantes la media de la diversidad sociocultural de los territorios de acción educativa.

En coherencia, se asume que es una condición para la interculturalidad generar desde la ETLBC espacios de relación entre jóvenes de distintos orígenes culturales y condiciones sociales. Con este propósito, y desde una perspectiva de gestión organizativa, es necesario profundizar en los factores que limitan la participación del conjunto de la diversidad sociocultural.

Estos factores se contextualizan en el sistema de interacción entre organizaciones educativas y comunidades locales. Y para su conceptualización deben tomarse en consideración referencias que vinculan el ámbito escolar con los procesos de concentración y segregación. Y que, además, señalan que estos procesos son obstáculos para la equidad educativa $y$, en consecuencia, para la interculturalidad.

Estas referencias se agrupan en tres claves:

- En clave de diversidad social: los procesos de concentración en el ámbito escolar se relacionan con dinámicas explicativas de desigualdad, materializándose en la concentración de grupos que son socialmente homogéneos (Alegre, 2013; Bonal, 2012). También variables como la condición social y las rendas familiares suelen ser determinantes para la elección de los centros escolares, concretándose en la configuración de procesos de segregación (Burgess, Wilson \& Lupton, 2005). En la ETLBC la combinación de estos elementos se refleja desde el carácter transversal de la diversidad social: los jóvenes de familias pertenecientes a rentas medias o altas suelen concentrarse en una tipología organizativa (esplais y scauts), y los jóvenes de familias de rentas más bajas en otras (centros abiertos).

- En clave de diversidad cultural: la diversidad cultural resulta un concepto complejo de analizar, especialmente si se considera que las categorías que la conforman se construyen socialmente, nutriéndose de elementos contextuales, subjetivos y dinámicos (Delgado, 1999; Giménez, 2012).

- En clave de complejidad: según lo señalado desde algunos trabajos, los procesos de segregación en el ámbito escolar suelen anteponerse a los procesos de concentración. Esto se debe a que se originan en el ámbito de la gestión organizativa y el ámbito privado de la decisión familiar. En consecuencia, esta relación plantea un escenario de complejidad que exige el análisis simultáneo de ambos procesos, puesto que se retroalimentan. Desde la ETLBC conviene incorporar al análisis la dimensión comunitaria, 
ya que parte de la base y acción de sus organizaciones educativas y se desarrolla con y para una comunidad específica. Además, en el ámbito de la investigación educativa, son extensas las referencias que abordan la relación entre segregación étnica y cultural, y segregación residencial desde una perspectiva del acceso (Bonal, 2012; Clotfelter, 1998; Reardon, Yun \& McNulty, 2000). Este elemento evidencia la importancia de considerar la dimensión comunitaria como elemento explicativo.

Partiendo de esta realidad, y de los resultados de un trabajo de investigación previo, en este artículo se presentan orientaciones para una gestión intercultural de los proyectos socioeducativos en el ámbito de la ETLBC. Las conclusiones pretenden contribuir a la superación de los procesos de exclusión educativos analizados anteriormente. $Y$ además se señalan futuras líneas de investigación para progresar en el estudio científico de este ámbito.

\section{Metodología}

La investigación en la que se contextualiza este artículo tenía como finalidad contribuir al fortalecimiento de interculturalidad en las organizaciones educativas de la ETLBC en Catalunya. Para ello, los objetivos de la investigación eran: 1) identificar condiciones organizativas y educativas que favorecieran o dificultaran el desarrollo de la interculturalidad, y 2) formular propuestas para su sostenibilidad o superación. A continuación, se detalla la metodología utilizada para lograr estos objetivos.

\section{Muestra}

La muestra del estudio estaba conformada por un total de 6 organizaciones educativas (2 organizaciones scauts, 2 esplais y 2 centros abiertos). Para su selección se utilizó un muestreo de conveniencia, no probabilístico y no aleatorio, según criterios de relación directa con los objetivos, siguiendo los fundamentos señalados por McMillan y Shummacher (2001). Los criterios utilizados para su confección fueron:

- Que los territorios donde se ubicaran las organizaciones educativas fueran considerados multiculturales, siendo al menos el $10 \%$ del conjunto de su población ciudadanía de origen extranjero;

- Que las organizaciones se ubicaran en la provincia de Barcelona;

- Que hubiera una distribución proporcional de organizaciones de base confesional y laica;
- Que los equipos educativos hubieran mostrado interés con el desarrollo intercultural.

Se incorporaron en la muestra los centros abiertos ya que, si bien no son definidos propiamente como organizaciones educativas de tiempo libre, comparten principios pedagógicos con la ETLBC (por ejemplo, su base dialógica o las actividades en grupo). Y, además, ofrecen servicios educativos propios de la ETLBC.

\section{Técnicas para la recogida de datos}

Siguiendo los requisitos metodológicos planteados por varios autores (Martínez, 2006; Kvale, 1996) se aplicaron técnicas cualitativas para la recogida de datos con una misma secuencia en la formulación de las cuestiones: dimensión descriptiva (¿cómo son las cosas?); dimensión axiológica (¿cómo quisiera que fueran?) y dimensión metodológica (¿qué se debe hacer para ello?).

Por un lado, se realizaron 31 entrevistas semi-estructuradas para incorporar y profundizar en percepciones y visiones de los entrevistados a partir de comentarios más vivenciales. Se entrevistaron: 12 miembros de equipos educativos, incluyendo miembros del equipo directivo; 11 jóvenes participantes de entre 15 y 18 años, con representatividad equitativa de ambos sexos, priorizando jóvenes de familia de origen extranjero (Marruecos, Bolivia, Congo, Argentina y Ecuador), aunque como elemento de contraste se entrevistaron también jóvenes de familia autóctona; y 8 miembros de las familias de estos jóvenes.

Y, por otro, se realizaron 2 grupos de discusión. El primero conformado por un miembro de cada equipo educativo de las organizaciones participantes, con el objetivo de analizar algunos de los resultados preliminares obtenidos con el análisis de las entrevistas. $Y$ el segundo, conformado por expertos en interculturalidad, para validar los resultados principales del trabajo de investigación.

También se analizaron 6 documentos aportados por las organizaciones educativas, para identificar continuidades o discontinuidades entre lo planteado normativamente y lo recogido desde las entrevistas.

\section{Procedimiento}

Para estructurar el proceso de recogida de la información se elaboró una matriz que permitió identificar y relacionar la fundamentación teórica del trabajo con los objetivos del mismo. Así, y desde los planteamientos ecológicos de Bronfenbrenner (1992), se distinguieron elementos facilitadores y dificultadores para el desarrollo 
intercultural desde un triple contexto: macro (ámbito general de la ETLBC), meso (proyecto educativo y equipo educativo) y micro (impacto de la interculturalidad en los participantes y visiones familiares). Esta matriz cumplió una función de orientación para la confección de los instrumentos, ya que fue elaborada al inicio del proceso de investigación, relacionando las dimensiones y ámbitos de estudio, las cuestiones clave a explorar y su relación con los instrumentos.

Para el tratamiento de los datos se realizó un análisis crítico del discurso. Se combinaron aportaciones de Van Dijk (2006), considerando que el discurso se relaciona con determinadas problemáticas sociopolíticas y desde la base de la desigualdad, y aportaciones de Calsamiglia y Tusón (1999), considerando su base más lingüística. Para el proceso de análisis de los datos se utilizó el programa Atlas-Ti realizándose una primera codificación temporal de 132 códigos. $\mathrm{Y}$, para una mejor gestión del alto volumen de datos introducidos, el análisis comprensivo se realizó desde una codificación definitiva de 35 códigos agrupados en 5 super códigos: organización, inclusión, exclusión, competencias interculturales y participantes. Todo esto dio lugar a la triangulación (teórica, metodológica y de informantes) final de los datos.

Para el análisis y presentación de los resultados se hizo referencia a dos tipos de escenarios educativos:

- Escenario "A": conformado por equipos profesionales de los Centros Abiertos. Tienen el encargo de desarrollar una atención integral combinando aspectos sociales, educativos y familiares.

- Escenario "B": conformado por equipos educativos voluntarios de esplais y scauts. Son elementos descriptivos de su finalidad tanto la participación comunitaria como la transformación social.

\section{Resultados}

A continuación, se recogen los resultados más significativos de la investigación presentada organizados en dos grandes apartados: aquellos resultados relativos a la organización; y aquéllos relativos a la comunidad.

\section{Relativos al ámbito organizativo}

En el ámbito organizativo de la ETLBC se evidencia la coexistencia de dos tipos de resultados. En primer lugar, aquéllos que favorecen el desarrollo de la inclusión educativa y el reflejo de la diversidad sociocultural del entorno desde los participantes. Y, en segundo lugar, aquéllos que son obstáculos para una gestión organizativa intercultural.

En relación a los primeros, es un elemento común y característico de estas organizaciones su visión amplia y activa de la participación. Esto se concreta en la existencia de distintas vías de acceso para que jóvenes y familias participen de los proyectos educativos:

- Desde el grupo de iguales cuando los jóvenes ejercen influencia ante sus iguales para invertir su tiempo de ocio en la ETLBC.

- Desde una decisión familiar cuando algún miembro familiar ya ha construido previamente un sentido educativo de la ETLBC, o bien a través del contacto con otra familia, o desde una experiencia previa de participación (especialmente familias autóctonas).

- Desde la orientación de otros servicios cuando centros educativos o servicios sociales valorizan en positivo los proyectos educativos de la ETLBC, orientando a los jóvenes para que participen.

También se concreta a partir de un planteamiento organizativo desde el cual los equipos educativos persiguen una máxima participación de nuevos jóvenes:

"Sí, tenemos lista de espera, pero podemos tener veinte niños en lista de espera como sesenta, que nunca tenemos suficiente, siempre buscamos y siempre intentamos abrir puertas" (fragmento de entrevista con educador/a, escenario educativo B).

Del mismo modo resulta una condición positiva que el conjunto de participantes describa la participación como significativa, positiva y densa. Además, estas valoraciones suelen ir acompañadas de la proyección de una idea de familiaridad, confianza y seguridad en la organización educativa:

"Soy una persona que puede ir por el mundo y que puedo respetar y creo que en parte es gracias a aqui" (fragmento de entrevista con participante, chica de 18 años, escenario educativo B).

Otro tipo de resultados favorables a la interculturalidad se sitúan en el desarrollo de algunas medidas de atención a la diversidad. Pero el conjunto de organizaciones no las impulsa simultáneamente, sino que su desarrollo es más bien puntual y fragmentado:

- Flexibilidad en la acogida de nuevos participantes: ofreciendo la oportunidad de un periodo de prueba para valorar la vinculación definitiva del futuro participante. Se orienta a familias de origen extranjero sin referentes 
previos de participación en la ETLBC y no supone coste económico añadido.

- Facilidades de pago para las cuotas periódicas: se aplica un sistema de ayuda económica ante dificultades familiares para el pago de las cuotas. Es significativo el incremento de estas situaciones a partir de la crisis económica actual:

“Lo importante es que estés aquí y si no puedes pagar nadie deja de venir aquí porque no lo puedas pagar. Esta reflexión la hicimos tarde, pero abrimos los ojos y desde que tenemos este nuevo discurso lo aprovechamos mucho" (fragmento de entrevista con educador/a escenario educativo B).

- Reflejo de la diversidad sociocultural en el propio equipo educativo: algunos equipos cuentan entre sus miembros con educadores pertenecientes a grupos socioculturales presentes en la comunidad. Con esta decisión organizativa se cuenta con más elementos para la comprensión de situaciones relativas a un contexto multicultural y se adquieren nuevas estrategias de comunicación con el entorno.

- Presentación del proyecto educativo a centros escolares: consiguiendo que nuevos miembros del profesorado conozcan el proyecto educativo y el sentido educativo de sus actividades. $Y$ que se interesen futuros participantes.

- Visibilización del proyecto educativo desde el espacio público: el objetivo es aumentar el número de participantes desarrollando actividades educativas en espacios públicos significativos para la comunidad en días específicos

En relación a las condiciones desfavorables al desarrollo de una gestión organizativa intercultural, destacan las resistencias dentro de los equipos educativos para impulsar medidas basadas en la discriminación positiva. Los equipos no son favorables a que perfiles socioculturales específicos de jóvenes accedan y participen de los proyectos educativos. Así, las organizaciones educativas del escenario " $\mathrm{B}$ " consideran que la discriminación positiva no es beneficiosa para el conjunto porque se ofrece un trato distinto sólo a una parte (futuros participantes de familia de origen extranjero) y esto genera desigualdades educativas. Por ello, se defiende un planteamiento igualitarista que considera necesario dispensar el mismo trato al conjunto de la diversidad sociocultural:

"Yo el momento en que se apuntan niños de otros orígenes no habrá ningún problema, pero por ejemplo no creo que se deba discriminar en este sentido, o sea como una discriminación positiva podríamos entender. En el sentido de nos reservamos tantas plazas para gente de culturas diferentes. Yo creo que esto es algo abierto y que hay unas inscripciones, se abren unas listas y cualquier persona que se apunte entrará, yo creo que ni por un lado ni por el otro se ha de discriminar" (fragmento de entrevista con educador/a, escenario educativo B).

Es también habitual que los equipos educativos refuercen estos planteamientos cuestionando la efectividad de estas medidas:

“Reservar unas plazas para unas personas que no sabes si vendrán y que quizás si no vienen hay un niño que sí quiera entrar y no lo pueda hacer, no sé yo... (...)" (fragmento de entrevista con educador/a, escenario educativo $B$ ).

Los equipos que sí muestran voluntad para desarrollar medidas de discriminación positiva expresan dificultades y falta de competencias que en la práctica imposibilitan su desarrollo:

"Queremos más niños, queremos convencer a más niños, vamos inventando cosas, a veces más acertadas y otras menos (...) pero de algún modo no sabemos dónde ir a buscar o como llegar" (fragmento de entrevista con educador/a, escenario educativo B).

Las organizaciones pertenecientes al escenario " $A$ " coinciden en expresar la imposibilidad para desarrollar este tipo de medidas. Señalan aspectos que superan su capacidad organizativa y no permiten asegurar que, desde sus participantes, se reflejen mayores cuotas de diversidad sociocultural. Por ejemplo, se antepone la exigencia de cubrir ciertas necesidades a contar con más diversidad sociocultural:

"Esto es una utopía que nosotros a día de hoy no valoramos, cuando tenemos listas de espera de muchísimos chavales, que no comen o solo tienen una comida al día, está muy bien, pero no llegamos" (fragmento de entrevista con educador/a, escenario educativo $A$ ).

Finalmente, es otra condición no favorable y común al conjunto de organizaciones, que sus equipos no se encuentren formados en interculturalidad. Si bien los equipos comparten la opinión de que la formación intercultural facilitaría el desarrollo de estrategias interculturales, en la práctica no se toman las decisiones necesarias para su concreción. 


\section{Relativos al ámbito comunitario}

A continuación, se presentan los tres tipos de resultados más significativos del ámbito comunitario, relacionados con: la existencia de procesos de sobre-representación sociocultural; la acción comunitaria de las organizaciones $y$, las visiones que se tienen de la diversidad familiar.

Determinados perfiles socioculturales de los participantes quedan sobre-representados si se comparan con la composición de la diversidad sociocultural del entorno. En concreto, el perfil de la participación desde el escenario " $A$ " muestra una proporción de jóvenes de familia de origen extranjero y de jóvenes en situaciones de vulnerabilidad socioeconómica muy superior a la existente en los territorios. A nivel numérico, la población de origen extranjero de los territorios que conforman la muestra se sitúa entre el $10 \%$ y el $40 \%$, pero el porcentaje de los participantes de familia de origen extranjero se sitúa entre el $60 \%$ y el $85 \%$.

En esta dirección se pronuncian miembros de equipos educativos:

"Aquí en la entidad hay población musulmana que no es representativa del barrio" (fragmento de entrevista con educador/a, escenario educativo $A$ );

"Nosotros atendemos a lo más necesitado, es un microcosmos que no representa la diversidad del barrio. Los Centros Abiertos deberían ser centros de integración de diferentes perfiles, de diferentes estratos, precisamente para no guetificar" (fragmento de entrevista con educador/a, escenario educativo A).

En cambio, la gran mayoría de participantes en las organizaciones del escenario " $\mathrm{B}$ " pertenece a familias autóctonas y a familias con una renta socioeconómica media o alta, y estos porcentajes duplican o triplican las proporciones presentes en los territorios. En estos términos lo expresa un/a educador/a:

"La gente que viene aquí generalmente pertenece a familias bien estantes y no tenemos ni diversidad ni multiculturalidad" (fragmento de entrevista con educador/a, escenario educativo B).

Destaca también que, ante el análisis de estos procesos de sobre-representación, los equipos educativos de ambos escenarios proyectan visiones de la diversidad sociocultural en las que aparecen elementos de homogeneidad y etnocentrismo. La siguiente respuesta se contextualiza en una organización en la que el $5 \%$ de sus participantes son de origen extranjero, pero en su territorio la población de origen extranjero representa el $20 \%$ del conjunto. La pregunta era si existe algún grupo, según origen cultural, significativo en el territorio, pero no presente en el proyecto educativo:

"No, yo creo que está todo" (fragmento de entrevista con educador/a, escenario educativo A).

Las visiones etnocéntricas se evidencian en muchos casos cuando se valora como problemática la concentración de jóvenes de familia de origen extranjero en proyectos educativos del territorio. Pero, al mismo tiempo, se valora en términos de normalidad cuando la concentración es de participantes de familia autóctona. De este modo, para el primer caso se utilizan adjetivos como "brutal" o "insostenible" en contraste con un lenguaje más descriptivo y neutral para referirse al segundo caso.

En relación a la acción comunitaria que se promueve, los equipos educativos de ambos escenarios no diseñan ni impulsan acciones conjuntas con la comunidad orientadas a la superación de los procesos de sobre-representación. Los equipos del escenario "B" manifiestan dificultades de coordinación con la comunidad educativa y, sobretodo, con los centros escolares, puesto que las colaboraciones son muy puntuales. Así mismo, los del escenario " $A$ " mantienen coordinaciones con miembros de la comunidad educativa para otras cuestiones, pero no se abordan aspectos relativos a la concentración o segregación educativa o a procesos de sobre-representación. En consecuencia, el conjunto de equipos educativos no construye una visión estratégica compartida con la comunidad educativa capaz de promover herramientas para una gestión intercultural de la diversidad sociocultural.

Y, por último, las visiones de los equipos educativos hacia la diversidad familiar no coinciden en muchos casos con las que proyectan las mismas familias hacia los equipos. Al respecto, el conjunto de familias - autóctonas y de origen extranjerovalora positivamente el trabajo educativo que se desarrolla:

"Lo único que puedo decir es que estoy muy agradecida, estoy encantada de venir aquí, mi hijo está feliz y contento, con eso ya soy la persona más feliz del mundo" (fragmento de entrevista con un miembro familiar de un participante, escenario educativo A).

No obstante, los equipos educativos muestran una visión homogénea de la diversidad familiar. Por ejemplo, se identifica una visión que enfatiza en ocasiones el déficit, invisibilizando potencialidades y recursos familiares: 
“Nosotros atendemos y lo que llega mayoritariamente, yo diría casi un $80 \%$ largo son familias con una situación socio económica muy complicada, difícil e impedimentos económicos y con una situación casi en la marginalidad. $Y$ entre unos que te hablo y no me entiendes y otros que no saben ni entienden por qué están aquí sus hijos (...)" (fragmento de entrevista con educador/a, escenario educativo $\mathrm{A}$ ).

A su vez, desde el escenario "B" se identifican unas expectativas hacia las familias de origen extranjero limitadoras, a partir de la percepción que no comprenderán el sentido educativo de la ETLBC. Sobre todo, cuando no se cuentan con experiencias de gestión educativa en contextos de diversidad:

“Esta gente inmigrante que entra yo creo que habría un punto de complicación en el sentido de no saber cómo funciona un esplai, de no saber los horarios, rutinas, costumbres, etc". (fragmento de entrevista con educador/a, escenario educativo B).

\section{Discusión y conclusiones}

Para cerrar este artículo se presentan algunas conclusiones que pueden orientar a la mejora, tanto desde el ámbito organizativo como comunitario, de todos los elementos trabajados.

\section{Relativas al ámbito organizativo}

Como conclusión general, conviene subrayar que las acciones organizativas que se desarrollan desde la ETLBC no son suficientes para la superación y transformación de los procesos de concentración y segregación educativa destacados recientemente (Síndic de Greuges, 2014). Si bien se identifican algunas condiciones que son favorables para reflejar más diversidad sociocultural entre los participantes, éstas quedan finalmente neutralizadas por diferentes obstáculos.

Desde el ámbito de la participación, resulta positivo que las organizaciones diversifiquen las vías para lograrla. De inicio se facilita que nuevos perfiles de jóvenes y familias conozcan y accedan a sus proyectos educativos. Este planteamiento inclusivo coincide con lo señalado por el informe del Síndic de Greuges de Catalunya (2014), que destaca la necesidad de fomentar el conocimiento y la valoración de la ETLBC por parte de colectivos socialmente desfavorecidos.

No obstante, resulta conveniente que los equipos educativos analicen y se posicionen acerca de si estas vías de participación son suficientes para lograr la participación de mayores cuotas de diversidad sociocultural. Son una minoría los jóvenes que conocen y participan de este contexto educativo, y lo son también los docentes que animan a que sus alumnos participen en él. Conviene subrayar que esta necesidad se contextualiza en organizaciones inclusivas, puesto que los equipos pretenden la máxima participación de jóvenes, y sus actuales participantes, con independencia de su perfil sociocultural, definen su participación en términos de significatividad y experiencia positiva.

Cabe destacar que las medidas de atención a la diversidad no se conciben como una prioridad, pues para los equipos educativos tampoco lo es reflejar mayores cuotas de diversidad sociocultural entre los participantes. Esta panorámica coincide con lo apuntado recientemente (Iglesias-Vidal, 2014; Síndic de Greuges, 2014) sobre la existencia de resistencias acerca de las medidas de discriminación positiva.

Por ello, su desarrollo responde a planteamientos reactivos ante situaciones específicas y no a planteamientos estratégicos y más bien proactivos. En concreto, la flexibilidad existente en la acogida de los participantes permite rebajar la incertidumbre a jóvenes que no cuentan con experiencias previas de participación en ETLBC. También es positivo que se faciliten de inicio elementos explicativos para una mayor comprensión del sentido educativo de la ETLBC. Además, la facilidad o exención de pago es también una medida inclusiva, ya que se orienta a que ningún participante deje de serlo por razones económicas. No obstante, conviene que estas acciones se contextualicen en un planteamiento organizativo más global configurado por las siguientes acciones:

- Diseñar y desarrollar procesos explicativos con el conjunto de la comunidad sobre el sentido educativo de su proyecto para que nuevas familias descubran que existen periodos de prueba y que las dificultades económicas no suponen un factor excluyente para la participación.

- Potenciar la diversidad cultural en los equipos educativos, incorporando nuevas competencias interculturales $y$, sobretodo, nuevas estrategias organizativas para una mayor comprensión del sentido de la participación familiar.

- Reformular el planteamiento que acompaña a presentar el proyecto educativo a los centros escolares y compartir el objetivo de contar con mayores cuotas de diversidad en la ETLBC.

- Visibilizar la organización educativa mediante actividades en el espacio público. Esto requiere un planteamiento estratégico, pues en la actualidad estas medidas no son del todo eficaces ya que no se acompañan de acciones complementarias como que un miembro del 
equipo educativo tenga la función exclusiva de comunicarse con las personas que observan las actividades. Resulta necesario resaltar la importancia de la comunicación presencial y directa entre equipo educativo y miembros de la comunidad (asociaciones de ciudadanos inmigrantes, centros escolares o familias). Todo ello permite trasladar, socializar y construir conjuntamente el valor educativo hacia nuevos perfiles socioculturales.

Finalmente, la falta de formación en interculturalidad de los equipos educativos plantea un amplio ámbito estratégico a recorrer y los equipos son conscientes de ello. Este resultado coincide con la necesidad señalada por diferentes autores (Generalitat de Catalunya, 2010; Herrera, Albaigés \& Garet, 2003; Iglesias-Vidal, 2014) de procesos formativos para que los equipos superen algunas de las dificultades a las que se enfrentan para el desarrollo de una perspectiva intercultural. De hecho, la falta de formación condiciona la construcción de significado que realizan de la diversidad sociocultural. Además, no se concibe como una prioridad organizativa, pues no se han formulado reflexiones internas que vinculen la superación de los procesos de concentración y segregación educativa con la dimensión organizativa y comunitaria de las organizaciones. Por ello, son comunes las resistencias de los equipos educativos a desarrollar medidas de discriminación positiva en este ámbito.

\section{Relativas al ámbito comunitario}

Partiendo de la idea de que la educación en el tiempo libre se contextualiza como educación comunitaria (Essomba, 2009), conviene fortalecer la dimensión comunitaria de los equipos educativos. Ello es condición para la gestión intercultural de la diversidad sociocultural. No obstante, el informe de la Generalitat de Catalunya (2010) señala algunas dificultades para este trabajo comunitario por particularidades de estas entidades como son el voluntariado y la elevada rotación de los educadores. El Síndic de Greuges de Catalunya (2014) también destaca algunas de las dificultades de coordinación en el ámbito comunitario.

A la luz de los resultados presentados aquí, resulta fundamental superar estas dificultades. Fortaleciendo la dimensión comunitaria de los equipos educativos se contribuye también a fortalecer el principio de prevención socioeducativa recogido en la finalidad de las organizaciones del escenario "A", y la capacidad de transformación social que define a las organizaciones del escenario " $B$ ".
Para ello es del todo necesario que los equipos educativos participen de espacios permanentes de análisis con la comunidad educativa. De hecho, la educación comunitaria prevé la participación de todos los miembros de la comunidad (familias, escuela y asociaciones infantiles y juveniles) (Essomba, 2009). En esta línea, debe seguirse un enfoque formativo-comunitario, abordando las siguientes tres líneas de trabajo:

- Reflexionar sobre las relaciones existentes entre la diversidad sociocultural presente en la organización educativa y la presente en el territorio de acción. Conviene identificar las causas por las que se establecen circuitos de acceso entre perfiles de participantes y organizaciones específicas. $Y$ también sus efectos en la configuración de procesos de concentración y segregación educativa en el conjunto del territorio.

- Identificar los vasos comunicantes que relacionan a centros escolares con la ETLBC a través de imágenes sociales y expectativas educativas sobre la diversidad sociocultural. Conviene tomar en consideración que estas imágenes y proyecciones tienen un impacto directo en: las expectativas personales y académicas que construyen los participantes; $y$, el tipo de adhesión educativa que construyen sus familias hacia los proyectos educativos de la comunidad.

- Descubrir los valores y sentidos educativos que construyen las familias sobre la ETLBC. Conviene distinguir aspectos singulares y relativos a los contextos culturales de origen, contando así con más elementos para la superación de una visión del déficit familiar. Los equipos educativos no visualizan la posibilidad de diversificar nuevos perfiles socio-familiares, ni han contrastado sus imágenes con otros miembros de la comunidad. Por ello, esta área de trabajo permitiría que los equipos sin experiencia educativa en contextos de diversidad incorporaran nuevos significados y expectativas sobre la diversidad familiar, apoyándose de la experiencia de gestión de otras organizaciones.

A modo de conclusión final, es recomendable la formulación de propuestas que combinen aspectos de los ámbitos organizativo y comunitario para contribuir a la superación de los procesos de concentración y segregación educativa abordados. Así, se propone, desde una perspectiva de gestión intercultural, la constitución de equipos comunitarios de atención a la diversidad, a partir de la implicación y alianza con administraciones y organizaciones educativas. 


\section{Limitaciones y futuras líneas de investigación}

La falta de antecedentes y referencias en este ámbito son entendidos como una limitación en esta investigación, puesto que no existen elementos de contraste para el avance en el proceso de investigación ni en la construcción de resultados y conclusiones. Así mismo, y como futuras líneas de investigación, se señala concretar, validar, desarrollar y evaluar un instrumento para el diagnóstico de la interculturalidad en este contexto educativo, y desde una perspectiva de desarrollo organizativo y comunitario.

\section{Referencias bibliográficas}

Abdallah-Pretceille, M. (2006). Lo intercultural como paradigma para pensar la diversidad. Actas Congreso INTER. Madrid: Servicio Publicaciones UNED.

Alegre, M.A. (2013). Elecció escolar: un breu marc de referència. En Tarabini, A. (Coord.), Les escoles magnet. Una aposta per l'excel.lència i l'equitat, Informes breus, 38, (pp. 25-32). Barcelona: Fundació Jaume Bofill.

American Association for Physical Activity and Recreation (2011). Leisure Education in the schools: promoting healthy lifestyles for all children and youth. Retrieved from: http://www.shapeamerica.org/advocacy/positionstatements/ loader.cfm?csModule=security/getfile\&pageid=5851.

Anderson, K., Caal, S., Carney, R., Lippman, L., Li, W., Muenks, K., Murphey, D., ... Terzian, M. (2014). Making the grade: assessing the evidence for integrated student supports. Retrieved from: https://www.childtrends.org/wp-content/ uploads/2014/02/2014-O7ISSPaper.pdf

Bartolomé, M. (2002). Introducción. Un reto a la educación intercultural. In M. Bartolomé (Coord.), Identidad y ciudadanía. Un reto a la educación intercultural (pp. 13-25). Madrid: Narcea.

Bonal, X. (2012). (Dir.). Municipis contra la segregació escolar. Sis experiències de política educativa local. Barcelona: Fundació Jaume Bofill.

Bronfenbrenner, U. (1992). Ecological systems theory. In Vasta \& Ross (Eds.), Six theories of child development: Revised formulations and current issues (pp. 187-249). London, England: Jessica Kingsley Publishers.

Burgess, S., Wilson, D., \& Lupton, R. (2005). Parallel Lives? Ethnic Segregation in Schools and Neighbourhoods Urban. Urban Studies, 42 (7), 1027-1056. doi: 10.1080/00420980500120741.

Calsamiglia, H., \& Tusón, A. (1999). Las cosas del decir. Manual de análisis del discurso. Barcelona: Editorial Ariel.

Clotfelter, C. (1998). Public school segregation in metropolitan areas. NBER Working Paper 6779. Cambridge: National Bureau of Economic Research.

Consejo de Europa (2008). Libro blanco sobre el diálogo intercultural. Vivir juntos con igual dignidad. Estrasburgo: Ministerio de Cultura. Gobierno de España.

Delgado, M. (1999). Anonimat $i$ ciutadania. Dret a la indiferència en contextos urbans. Barcelona: Institut Català d'Antropologia

Essomba, M.A. (2009). De l'educació en el lleure a l'educació comunitària. Avançant cap a la inclusió social. In Educació Comunitària, Col-lecció Parlem-ne (pp. 57-74). Barcelona: Minyons Escoltes Guies Sant Jordi de Catalunya.

Essomba, M.A. (2011). Inmigración e interculturalidad en la ciudad. Principios, ámbitos y condiciones para una acción comunitaria intercultural en perspectiva europea. Barcelona: Graó.

Fundació Ferrer i Guardia (2013). Els esplais en xifres. La realitat d'esplais catalans. Barcelona: ESPLAC.

Generalitat de Catalunya (2010). Mesures de foment, reconeixement $i$ suport de l'educació en el lleure de base comunitària. Barcelona: Departament d'Acció Social i Ciutadania. Secretaria de Joventut.

Giménez, C. (2012). Pluralismo, multiculturalismo e interculturalidad: ¿qué significa "intercultural" cuando hablamos de “educación intercultural”? In L., Díe (Coord.). Aprendiendo a ser iguales. Manual de educación intercultural (pp. 48-65). Valencia: Ceimigra.

Herrera, D., Albaigés, B., \& Garet, M. (2003). La immigració i el treball intercultural a les entitats d'educació en el lleure a Catalunya. Barcelona: Generalitat de Catalunya. Departament de la Presidència. Secretaria General de Joventut.

Iglesias-Vidal, E. (2014). La formación en interculturalidad en la educación en el tiempo libre: necesidades y estrategias desde una perspectiva de competencias interculturales. Revista Electrónica Interuniversitaria de Formación del Profesorado, 17 (2), 167-182.

Kvale, S. (1996). Interviews: An introduction to qualitative research. Londres: Sage publications.

Martínez, M. (2006). La investigación cualitativa (síntesis conceptual). Revista IIPSI. Facultad de Psicologia, UNMSM, 9 (1), 123-146.

McMillan, J., \& Shummacher, S. (2001). Research in education: A conceptual introduction (5ta ed.). New York: Addison Wesley Longman. 
Reardon, S., Yun, F., \& McNulty, T. (2000). The changing structure of school segregation: measurement and evidence of multiracial metropolitan-area school segregation, 1989-1995. Demography, 37 (3), 351-364. doi: 10.2307/2648047.

Sales, A. (2011). La formación intercultural del profesorado: estrategias para un proceso de investigación-acción. Educatio Siglo XXI, 30 (1), 113-132.

Síndic de Greuges (2014). Informe sobre el dret al lleure educatiu i a les sortides i colònies escolars. Barcelona: Síndic, el defensor de les persones.

Vallory, E. (2010). L'escoltisme mundial. Barcelona: Proa.

Van Dijk, T. A. (2006). Discurso, poder y cognición social. Conferencias de Teun A. Van Dijk. Maestría. Cuadernos 2 (2). Retrieved from http://www.discursos.org/oldarticles/Discurso,\%2Opoder\%2Oy\%2Ocognici\%F3n\%2Osocial.pdf

Wood, P., \& Landry, C. (2008). The intercultural city. Planning for diversity advantage. Londres: Earthscan.

Xarxa de Centres Oberts de Barcelona (s.f.). Els centres oberts a la ciutat de Barcelona. Barcelona: Ajuntament de Barcelona.

Zapata-Barrero, R., \& Pinyol, G. (Eds.). (2013). Manual para el diseño de políticas interculturales. Policy Series,1. Barcelona: GRITIM-UPF.

\section{Notas}

1 Este contexto organizativo se define desde su carácter de atención social pero también promueve servicios propios de la educación del tiempo libre.

2 Traducción: "Los programas educativos inclusivos en el tiempo libre son necesarios para todas las personas, especialmente para individuos y grupos que han sido oprimidos y pueden encontrar en las actividades de ocio restricciones por razón de género, raza, étnica, capacidad, edad, orientación sexual, ingresos, condiciones de vida, religión y otras características destacadas".

\section{CÓMO CITAR ESTE ARTÍCULO}

Iglesias-Vidal, E., \& Espona-Barcons, B. (2017). Propuestas para el desarrollo de la interculturalidad en la educación en el tiempo libre de base comunitaria en Catalunya. Pedagogía Social. Revista Interuniversitaria, 30, 145-157. DOI: 10.7179/PSRI_2017.30.10.

\section{DIRECCIÓN COMPLETA DE LOS AUTORES}

Edgar Iglesias-Vidal: Universidad Autónoma de Barcelona (UAB), Departament de Pedagogia Aplicada. Edifici G6-255. Campus de Bellaterra. 08193, Bellaterra (Cerdanyola del Vallès). Grupo de Investigación ERDISC. Edgar.iglesias@uab.cat

Berta Espona-Barcons: Universidad Autónoma de Barcelona (UAB), Grupo de Investigación ERDISC. bertaespona@gmail.com 
PERFIL ACADÉMICO

Edgar Iglesias-Vidal: Actualmente y desde el 2008 es profesor asociado al Departamento de Pedagogía Aplicada de la UAB. Es también miembro del área técnica de la Asociación Educativa Integral del Raval en Barcelona. Es Doctor en Pedagogía por la UAB en el 2014 y Premio extraordinario de investigación de la misma universidad, por su tesis doctoral "La educación intercultural en las organizaciones educativas de base comunitaria". Se licenció en Antropología Social y Cultural en el 2007 por la UAB y fue Visiting Scholar en el 2016 por la Graduate School of Education de la Stanford University (California - EEUU). Fue becado por el Consortium for Advanced Studies in Barcelona para desarrollar una investigación en educación comunitaria en California. Ha realizado distintas investigaciones y publicaciones, publicando recientemente el libro "Educación intercultural en el tiempo libre" (Ed. Popular 2015) y cuenta con más de 18 años de experiencia en la intervención socioeducativa. A su vez, asesora y colabora en la actualidad con diferentes equipos educativos.

Berta Espona-Barcons: Licenciada en Pedagogía por la UAB y Máster de Investigación en Sociología Aplicada. Ha trabajado como técnica superior de investigación en los departamentos de Pedagogía Aplicada y Pedagogía Sistemática y Social de la UAB des del año 2010. Des del 2008 ha sido monitora voluntaria en asociaciones de educación en el tiempo libre de base comunitaria. $\mathrm{Y}$ ha trabajado en diferentes proyectos socioeducativos comunitarios en este ámbito. Del 2012 al 2014 gestionó un proyecto europeo sobre política educativa e inmigración. Actualmente trabaja como tutora y formadora de cursos de monitor y monitora de educación en el tiempo libre. Algunos de los jóvenes que dinamiza se encuentran en situación de vulnerabilidad social. Y continúa colaborando con diferentes proyectos de investigación. 
